\title{
ELEMENTOS QUE MOTIVAN AL USUARIO A OBTENER CERTIFICACIÓN DE "CURSOS EN LÍNEA" EN EDUCACIÓN SUPERIOR
}

\section{ELEMENTS THAT MOTIVATE USERS TO OBTAIN CERTIFICATION OF "ONLINE COURSES" IN HIGHER EDUCATION}

\author{
Natalia Castañón Octavio. Universidad Metropolitana. Venezuela.
}

\section{RESUMEN}

Debido al aumento mundial del interés en los cursos en línea, toma importancia adaptar los cursos de Unimet en línea a las necesidades de sus usuarios. El objetivo de este estudio es determinar los elementos que motivan a los usuarios a obtener certificaciones de cursos en línea en Educación Superior. Lo anterior se logra mediante el instrumento de una encuesta a 899 usuarios de los 11.000 inscritos en la plataforma Unimet en línea para el momento del estudio. Las respuestas recibidas indican que la mayor parte de los que realizan cursos son personas con grado de licenciatura. Se observa que una mayoría del $80 \%$ no adquirió certificados en la plataforma, debido a razones de índole monetaria, de relevancia y la no culminación del curso. Se puede decir que los usuarios le han dado mayor importancia a los conocimientos que a la obtención del certificado. Mediante el estudio se logró una perspectiva más amplia de los elementos estudiados, que permiten ajustar los cursos para promover la certificación de los usuarios.

PALABRAS CLAVE: MOOCs, motivación, cursos, e-learning, educación a distancia.

\section{ABSTRACT}

Due to the worldwide increase of interest in online courses, it is important to adapt Unimet en línea's courses to the needs of its users. The objective of this study is to determine the elements that motivate users to obtain certifications of online courses in higher education. The above is achieved through the instrument of a survey to 899 users of the 11,000 enrolled in the online Unimet platform for the time of the study. The answers received indicate that most of those who take courses have a bachelor's degree. It is observed that a majority of $80 \%$ did not acquire certificates in the platform, due to reasons of monetary nature, relevance and non-completion of the course. It can be said that users have given greater importance to knowledge than to obtain the certificate. Through the study, a broader perspective of the elements studied was achieved, which allows adjusting the courses to promote user certification.

KEY WORDS: MOOCs, motivation, courses, e-learning, long distance education.

\footnotetext{
${ }^{1}$ Natalia Castañón Octavio: Universidad Metropolitana / Departamento de Ciencias de la Educación

/ Caracas / Venezuela.

ncastanon@unimet.edu.ve
} 
Castañón Octavio, N. (2018). Elementos que motivan al usuario a obtener certificación de "cursos en línea" en educación superior. Revista de Ciencias de la Comunicación e Información, 23(1), 1-14.

doi: http://doi.org/10.35742/rcci.2018.23(1).1-14

\section{INTRODUCCIÓN}

La formación en línea, es una capacitación que utiliza la red como tecnología de distribución de la información. En otras palabras, formación en línea (en ocasiones denominada e-learning o educación virtual) es aquella en la que los docentes y estudiantes participan en un entorno digital a través de las nuevas tecnologías y de las redes de computadoras, haciendo uso intensivo de las facilidades que proporciona Internet y las tecnologías digitales. Y para profundizar esta breve definición, Schell (como se citó en Cabero, 2006, p. 2) expone que "los cursos de formación en red son definidos para nuestro propósito como cursos donde la mayoría, si no toda, de la instrucción y de las pruebas se logran vía recursos accesibles en la Web".

Las Instituciones de Educación Superior han sido las que mayoritariamente han adoptado la modalidad virtual dentro de sus diseños y modelos educativos. Para ello, Suárez (2016) indica que actualmente no existe algún modelo educativo de Universidad ajeno a Internet y gran parte de las actividades creativas y rutinarias de la mayoría de las universidades están mediatizadas por un entorno tecnológicamente enriquecido que permite pensar y hacer las cosas de una forma global y en red.

Por ende, la mayoría de las universidades a nivel mundial (aproximación de un 70\%) considera la integración de las TIC en el proceso de enseñanza-aprendizaje, tomando en cuenta el contexto de la evolución de la sociedad, del cambio social, etc. Incluso tiende a ser analizada como una oportunidad de "mercado" que puede mejorar a la institución, ya que suelen aparecer nuevas posibilidades virtuales como alternativas a las convencionales.

\section{OBJETIVOS}

\subsection{Objetivo general}

Determinar los elementos que motivan al usuario a obtener certificación de "cursos en línea" en Educación Superior.

\section{METODOLOGÍA}

\subsection{Diseño de la Investigación}

Kerlinger (1979) expone que "La investigación no experimental o expost-facto es cualquier investigación en la que resulta imposible manipular variables o asignar aleatoriamente a los sujetos o a las condiciones" (p. 116). Por otra parte, cabe 
destacar, que las investigaciones no experimentales, exponen dos tipos sobre los cuales el investigador se puede basar, y uno de estos tipos es el transeccional, ya que "se centra en analizar cuál es el nivel o estado de una o diversas variables en un momento dado o en cuál es la relación entre un conjunto de variables en un punto en el tiempo" (Dzul, 2013, p. 5).

Por lo tanto, el diseño de la presente investigación corresponde a un estudio no experimental de tipo transeccional, debido a que se realizará sin la manipulación deliberada de las variables, es decir, únicamente serán observados los fenómenos que inciden en ellas en su ambiente natural, basándose en la recopilación de datos realizados en un momento único.

\subsection{Tipo de Investigación}

El tipo de investigación será de carácter descriptivo, ya que la misma se basará en la indagación, registro y definición de la experiencia del usuario dentro de la plataforma de Unimet en Línea. Para esto, la investigación se apoyará en la construcción de instrumentos de medida válidos y confiables, partiendo del conocimiento previo teórico a fin de medir de forma independiente las variables de la investigación sin establecer relaciones causa-efecto.

Además, es importante recalcar que "su meta no se limita a la recolección de datos, sino a la predicción e identificación de las relaciones que existen entre dos o más variables" (Van Dalen y Meyer, 2006). Donde los investigadores, deben encargarse de recoger los datos sobre la base de una teoría, exponer y resumir la información de manera cuidadosa y luego analizan minuciosamente los resultados, con la finalidad de extraer generalizaciones significativas que contribuyan al conocimiento.

De igual modo, para obtener una impresión general de la experiencia de la población dentro de la Plataforma Unimet en Línea, se llevará a cabo un estudio tipo encuesta, el cual permitirá reflejar mediante encuestas la tendencia de opinión de los usuarios, sin entrar en motivos individuales.

\subsection{Variables}

Según Daniel Cauas (s.f.):

El término está tomado de las matemáticas, utilizándose de forma bastante elástica en el ámbito de las ciencias sociales. Por lo general, se utiliza como sinónimo de "aspecto", "propiedad" o "dimensión". Propiedad o característica de un objeto, o fenómeno que presenta variaciones en sucesivas mediciones temporales (p. 3).

"Las variables, resultado del proceso de operativización desde el plano teórico al plano empírico, son las manifestaciones de los constructos, y a las que se les puede asignar valores o palabras, que el investigador va a relacionar o constatar" (Buendía, Colás, Hernández, 2001, p. 2).

En otras palabras, las variables corresponden a los atributos o características deliberadas por el investigador, cuyos valores pueden ser medibles u observables. 
Es decir, se trata de una propiedad del objeto de estudio la cual se encuentra ligada a otros aspectos observables.

Asimismo, tal como expone el documento de la Universidad Andrés Bello "Tema: Las Variables" (s.f.), según el criterio metodológico pueden ser divididas en: dependientes e independientes.

\subsubsection{Variables dependientes}

Son aquellas cuyos valores varian en función de otras variables. (Hernández, Fernández y Baptista, 2003).

\subsubsection{Variables independientes}

Corresponden a aquellas propiedades del objeto de estudio que el investigador manipula y/o mide para ver los efectos que produce sobre otra variable. (Hernández, Fernández, 2003).

\subsection{Población y Muestra}

La población es entendida como el "conjunto finito o infinito de elementos con características comunes, para los cuales serán extensivas las conclusiones de la investigación. Esta queda limitada por el problema y por los objetivos del estudio". (Arias, 2006. p. 81). Entonces, al considerar que la población es la totalidad del fenómeno a estudiar, donde las unidades de población poseen una característica común, y que debe ser estudiada para así dar origen a los datos de investigación; se puede definir que la población a trabajar en la presente investigación corresponde a todos aquellos usuarios que se encuentran inscritos en la plataforma Unimet en Línea.

La muestra, es lo que puede determinar la problemática de la investigación, ya que la misma es capaz de generar los datos con los cuales se identifican las fallas dentro del proceso. Según Tamayo y Tamayo (1997), afirma que la muestra "es el grupo de individuos que se toma de la población, para estudiar un fenómeno estadístico" (p.38).

De igual modo, es importante recalcar la existencia de dos tipos de muestra, los cuales son: aleatoria e intencional, donde el tipo de muestreo utilizado para la presente investigación será la aleatoria, ya que sin previa selección se tomarán en cuenta las opiniones de todos los usuarios que hayan decidido contestar el instrumento, el cual permitirá arrojar resultados factibles y de gran necesidad a la investigación.

Ahora bien, al conocer que la población al momento de enviar el instrumento será de 11.000 usuarios, según lo comentado por el Equipo de Unimet en Línea a las investigadoras. Entonces, para garantizar 95\% de nivel de confianza de la muestra, con un margen de error de $5 \%$ será necesario obtener 372 encuestas respondidas, según los cálculos realizados para definir tamaños de muestra con conocimiento del tamaño de la población. 


\subsection{Instrumento de Recolección de Datos}

Esta fase de la investigación, consiste en el proceso de recopilación de información necesaria para el estudio del fenómeno seleccionado.

En la presente investigación, como instrumento de recolección de datos se utilizará la encuesta. Ya que ésta consiste en un cuestionario que permite detectar ideas, necesidades, preferencias, hábitos de uso, que sin duda corresponderá a insumos indispensables para cumplir con el objetivo del presente trabajo. Y adicionalmente, es un instrumento que permite ser aplicado en una muestra numerosa y dispersa. (Cerda, 1991)

Asimismo, García, citado por Torres, Paz y Salazar (s.f., p. 4), define la encuesta como:

Una investigación realizada sobre una muestra de sujetos representativa de un colectivo más amplio, utilizando procedimientos estandarizados de interrogación con el fin de obtener mediciones cuantitativas de una gran variedad de características objetivas y subjetivas de la población.

Para esto, se utilizará el portal web "Encuesta Fácil", el cual permite diseñar un instrumento ajustado a las características de la presente investigación y tiene la virtud de compartir la encuesta a través de un link. Por lo tanto, a medida que los usuarios vayan contestando se podrá tener una visión general de la tendencia de los resultados.

La encuesta será compartida a través del email de Unimet en línea (enlínea@unimet.edu.ve), las veces que sea necesario para conseguir el número de encuestas respondidas que cumpla con el tamaño de muestra representativa, calculado previamente.

\subsection{Análisis de Datos}

Luego de haber recopilado los datos de investigación, se procederá a realizar el análisis de los mismos. Dicho en otras palabras,

Una vez recopilados los datos brutos el proceso de su análisis y teorización requiere organizarlos previamente, lo cual puede hacerse a través de: selección, focalización, abstracción y transformación de ellos a partir del uso de códigos, matrices, redes y/o mapas que permitan la mejor y mayor visualización de las características comunes y no comunes de estos datos. (Vegas, Calderón y Rodríguez, 2005, p. 4).

Para ello, la investigación se apoyará en el análisis cualitativo y cuantitativo. Ya que en el primer caso, permitirá realizar una interpretación profunda de las realidades y de su estructura dinámica basándose en los aspectos subjetivos (Mendoza, 2006), cómo será el caso de algunas preguntas abiertas en la encuesta a realizar. Adicionalmente, se utilizará el análisis cuantitativo a fin de proyectar a través de cifras numéricas y métodos estadísticos los resultados arrojados en las encuestas (Mendoza, 2006). 


\section{DISCUSIÓN}

\subsection{Tecnología y educación}

Actualmente, para nadie resulta novedosa la idea de que somos un planeta que se encuentra plenamente inmerso en los avances tecnológicos, y que la sociedad debe ir de la mano de estos avances, para así lograr mayores innovaciones y no quedarse en una sociedad arcaica o antigua. Y quien más ha estado de la mano de estos avances, ha sido la Educación Superior, pues a nivel mundial la misma ha incorporado la virtualidad en sus modelos educativos, ya que la nueva educación "necesita nuevos paradigmas que han sido previamente distinguidos por diversos pedagogos. Todos ellos promovieron una educación más libre, más centrada en el estudiante, sus necesidades y ritmos de aprendizaje, más individualizada, interactiva, cooperativa, participativa y constructiva" (Silvio, 2004, p. 5).

Según Fredrickson (1999), citado por Román Esperanza (2000), la evolución de plataformas para el desarrollo de cursos en la Web ha tenido un "efecto de bola de nieve", donde la propagación de cursos online ha provocado la rápida aparición de plataformas, lo que a su vez está causando la elaboración de más cursos. Tanto así, que en la actualidad es fácil decir que existen aproximadamente más de 100 plataformas educativas a nivel mundial y son utilizadas por tantas personas, que en una sola plataforma, la cual es la más reconocida (Moodle) tiene más de 72 millones de personas inscritas (Moodle, 2015).

\subsection{Formación en línea en educación superior}

Entre el año 2002 y 2003, el IESALC (Instituto Internacional de la Unesco para la Educación Superior en América Latina y el Caribe), realizó un proyecto de investigación cuyos objetivos fueron conocer la evolución, situación y perspectivas de la educación superior virtual en América Latina y el Caribe, específicamente se efectuaron dos estudios de cobertura subregional, uno en Centroamérica, otro en el Caribe Anglófono y 13 estudios nacionales en los siguientes países: Argentina, Bolivia, Brasil, Colombia, Chile, Ecuador, México, Perú, República Dominicana, Uruguay y Venezuela. De acuerdo a los resultados, Silvio (2004) destacó que:

Se identificaron 175 instituciones con 11 programas en marcha de la modalidad de educación virtual, que representan un $20,7 \%$ del total de las instituciones. Además, se identificaron 164.527 estudiantes cursantes de programas de educación virtual. $\mathrm{Y}$ en el conjunto se destacan Brasil, con 84.000 estudiantes y México con 30.000. (p. 10).

Por lo tanto, es notorio el gran avance que ha logrado la educación superior, en cuanto a la aplicación de la tecnología en sus métodos de enseñanza; que si bien es cierto el proyecto arrojó bajo resultados, se debe tomar en cuenta que dicho proyecto fue aplicado hace 12 años.

Por otro lado, en el año 2007, Bernárdez realiza un trabajo de investigación, en el cual expone el Ranking de América para e-Learning, en donde los valores fueron analizados como "usuarios por cada 10.000 habitantes", y como resultados arrojados obtuvo: Estados Unidos en primer lugar con 5.513 usuarios, Canadá con 5.128 
usuarios, Chile con 2.719, México con 1.184, Argentina con 1.120, Perú con 1.039, y por último Brasil con 822 usuarios. Ahora bien, tal como era de esperarse con el pasar de los años la tecnología invade cada día más al área educativa, donde es evidente cómo las instituciones buscan aplicar la formación en línea o enseñanza virtual en sus diseños curriculares.

El conocimiento se encuentra al alcance de un clic, ha llegado a todos los rincones del mundo gracias a la tecnologías, esto ha implicado que los sistemas educativos creen espacios para contribuir en la distribución del conocimientos, por medio de los espacios virtuales de aprendizaje, ya que permiten organizar los conocimientos en contenidos y dar respuesta al llamado de educación permanente hace el mercado laboral.

Ahora, el vuelco que ha dado la educación y el uso de plataformas educativas, han permitido facilitar los procesos de aprendizaje, ya que permiten al estudiante participar, compartir, intercambiar y producir contenidos propios mediante las herramientas sencillas que ofrecen los espacios virtuales de aprendizaje (Dans, 2009).

A continuación, se presentan algunos datos que revelan de cómo la tecnología ha impactado en los modelos de aprendizaje, y por lo tanto, en los procesos de formación y capacitación de los individuos en la actualidad. Los resultados de los estudios de investigación de Aurion Learning (2015) y The Training Zone (2015) destacan datos que revelan las tendencias actuales de la educación en línea, tales como:

- $\quad 42 \%$ de las compañías líderes especializadas en educación han reconocido que el aprendizaje en línea ha sufrido un incremento en ganancias.

- $81 \%$ de los involucrados en algún proceso de aprendizaje en línea están complacidos de manejar su propio desarrollo personal.

- Las organizaciones que han dado importancia al aprendizaje y desarrollo profesional de sus empleados tienen 13 veces más posibilidades de obtener ganancias.

- 3 de cada 5 organizaciones no pueden implementar grandes cambios en sus procesos debido a la falta de competencias actualizadas de sus empleados.

- $49 \%$ de las organizaciones cuentan con una planta de empleados actualizados en las competencias que necesitan.

- En el 2015 el 98\% de las organizaciones recurrirá al uso de cursos en línea como parte del proceso de entrenamiento y desarrollo profesional de sus empleados.

- Para 2016 más del 90\% de las organizaciones empleará videos como parte de sus estrategias de aprendizaje en línea.

- El $74 \%$ de las personas emplean actualmente algún dispositivo móvil para educación en línea.

- Actualmente el $42 \%$ del sector público emplea MOOCs para lograr sus metas de desarrollo.

- En el 2019 el 50\% de las clases en todos los niveles educativos será realizada en línea. 
Estos datos deben ser capitalizados y comprendidos por las instituciones de educación superior y responder a las necesidades del entorno. Es por ello que la Universidad Metropolitana propone el proyecto Unimet en Línea, inicialmente como una plataforma educativa de formación en línea, dirigida a hispanoparlantes y gratuita.

\subsection{MOOCs}

Los Open Educational Resources (OER) (Recursos Educativos de Acceso Abierto) tienen el potencial para ampliar el acceso a la educación y mejorar la calidad y rentabilidad de la enseñanza y aprendizaje. El método más exitoso para lograr una implementación práctica de OERs son los Massive Open Online Courses (MOOCs) (Cursos masivos en línea de acceso libre o abierto). Campus virtual es un espacio para la enseñanza, aprendizaje e investigación creado mediante la confluencia de múltiples aplicaciones de las TIC: Internet, Web, comunicación electrónica, video, video-conferencia, multimedia y publicaciones electrónicas (Van Dusen, 1997). Estos espacios suelen también denominarse aula virtual, universidad en línea (on-line), eCampus, eUniversidad, ciberaula y campus tecnológico. Los sistemas de información y proceso utilizados como soporte de un campus virtual se denominan con el término genérico Managed Learning Environment (MLE). Los MLEs más habituales son los Learning Management Systems (LMSs). Los MLEs son herramientas que facilitan a los profesores la gestión y administración integrada de los cursos en que imparten docencia. Incluyen, normalmente un entorno web, funciones y planificación de aprendizaje, gestión y seguimiento de los alumnos, comunicación sincrónica, asincrónica, y la publicación de materiales docentes (Fernández-Valmayor, Cristobal, Navarro, Fernández, Merino, Peralta, y Roldán, 2008). Para este estudio se hizo una revisión exhaustiva de los LMSs actuales y las decisión estuvo basada más en variables pedagógicas que en las tecnológicas como se reflejará en los resultados.

Ahora bien, en este marco contextual no se puede obviar los Moocs que han despertado un gran interés durante estos últimos años dentro del mundo docente. Los MOOCs se definen como cursos en línea de acceso abierto y dirigidos a un número ilimitado de participantes (Gómez, 2014). Estos se caracterizan por ser cursos en línea, sin requisitos de acceso formales, de difusión masiva, sin límite de participantes, usualmente sin coste para los inscritos en caso que el usuario no esté interesado en el certificado. Los MOOCs son una tendencia que consideran tres aspectos: acceso masivo, producción del curso y viabilidad financiera. Este artículo expondrá las opciones consideradas al respecto.

\subsection{Unimet en Línea}

La Universidad Metropolitana, no se ha quedado atrás en ofrecer educación de vanguardia en espacios virtuales. Es por esto, que siguiendo el modelo educativo de la institución diseñó la plataforma Unimet en Línea.

Allí, la Universidad ofrece formación gratuita en línea a todos los habitantes de Latinoamérica y el Caribe, que deseen desarrollarse tanto académica, como profesional, y personalmente, a través de cursos elaborados por profesionales de alto nivel. (enlinea.unimet.edu.ve). 
La manera en que se encuentra diseñada la plataforma, y la organización de los contenidos dentro de los cursos, permite al usuario gozar de ciertas ventajas que nutrirán su proceso de formación, como: apoyo y seguimiento del profesor, innovación pedagógica, aprendizaje autónomo y auto-administrado, dinámico, interactivo y con variedad de recursos en línea.

Por otro lado, es imprescindible destacar que luego de aprobar cualquiera de los cursos, el usuario puede optar por obtener un certificado avalado por la Universidad Metropolitana, el cual luego de realizar el pago electrónico, será enviado a su cuenta de email.

Unimet en Línea, es un espacio virtual donde se ofrecen cursos, dictados por profesionales de la docencia del más alto nivel, que permiten al usuario capacitarse en temas específicos y desarrollarse a nivel académico, profesional y personal (enlinea.unimet.edu.ve). Adicionalmente, cabe destacar que todos los cursos ofrecidos en la plataforma Unimet en Línea son avalados por la Universidad Metropolitana por medio de certificados, por los cuales los usuarios pueden optar.

\section{RESULTADOS Y CONCLUSIONES}

Se obtuvieron 899 encuestas respondidas lo cual sobrepasó el número requerido (372 sujetos) para conseguir el tamaño de una muestra representativa de $95 \%$ de confiabilidad con $5 \%$ de error. Con 610 respuestas como mínimo, se recalculó con ayuda del portal Netquest los niveles de error y confiabilidad de la encuesta. Los cálculos arrojados por el portal fueron de $4 \%$ de error y $95 \%$ de confiabilidad. Los resultados más destacados son:

- De acuerdo a las 899 respuestas recogidas, se aprecia que el $64 \%$ de las personas encuestadas son mujeres, mientras que tan solo el $36 \%$ son hombres, específicamente estos porcentajes corresponden a 571 mujeres y 328 hombres. Con respecto a la edad de los usuarios encuestados de Unimet en Línea, es posible afirmar que la mayoría de estos se encuentran en un rango de edad mayor de 25 años. Unimet en Línea, es una plataforma enfocada principalmente a dos grupos: individuos profesionales mayores de 25 años y empresas. El rango de edad donde se encuentran la mayoría de los usuarios es mayor de 46 años.

- De las 883 respuestas recogidas, se aprecia que un número importante de 740 corresponden a usuarios venezolanos, liderando este la lista de las nacionalidades. En segundo lugar, se encuentran 63 personas de nacionalidad mexicana. Posteriormente, se resaltan las nacionalidades: colombiana (23), española (18), peruana (15), chilena (11), argentina (6) y ecuatoriana (6).

- En base a las 881 respuestas recogidas, se evidencia que un $48 \%$ de las personas son licenciadas, el $20 \%$ son magister, el $15 \%$ son técnico, el $14 \%$ bachiller y por último el $3 \%$ doctor. Por ende, se corrobora lo expuesto por Castañón y Del Valle (2015) en su estudio mencionado en el capítulo Unimet en Línea, donde la motivación principal de los usuarios profesionales para realizar cursos es poder conseguir mejoras en el ámbito laboral. 
- Los estudiantes (116) es la ocupación predominante entre los usuarios que contestaron esta pregunta, seguidos por los docentes (114), ingenieros (52), administradores y abogados, cada uno con un total de 51 personas. Luego, se destacan las ocupaciones de: psicólogo (32), gerente (30), consultor (20), diseñador (16) y contador (15).

El $80 \%$ de ellos no adquirieron los certificados. Las razones son:

- De acuerdo a las 502 personas que dijeron que no compraron el certificado, $27 \%$ se refirió a que esto se debía al elevado costo del mismo. Por otro lado, el $20 \%$ opinó que no lo consideraban relevante. Por último, el mayor número de los usuarios que respondieron la pregunta (52\%) seleccionaron la opción "Otro", donde especificaron sus razones.

- Las 263 personas que seleccionaron la opción "otro", indicaron entre sus razones por las cuales no compraron el certificado que aún no han culminado el curso (203), abandonaron el curso (13), en proceso de pago (30), dificultades referentes al pago presentadas en el sistema (5) y desacuerdo con la forma de pago del certificado (8). En vista de los es posible inferir que los usuarios le están dando mayor importancia a la adquisición de conocimientos a través de los cursos de la plataforma, que al certificado en sí.

- Por otro lado, con respecto a las 130 personas que sí adquirieron el certificado, el $90 \%$ de ellas seleccionaron las 2 calificaciones máxima, es decir, 5 y 4 . Refiriéndose al proceso de certificación como sencillo y amigable. Sin embargo, el $5 \%, 1 \%$ y $4 \%$, seleccionaron los puntajes más bajos.

- Asimismo, el 89\% opinó que el pago del curso fue sencillo; mientras que, el $12 \%$ lo calificaron con los puntajes; 3,2 y 1. Demostrándose, una diferencia significativa entre las opiniones de los usuarios, donde la opinión de la mayoría favorece a la plataforma Unimet en Línea.

- Por último, el $87 \%$ consideró que la impresión del certificado fue rápida y fácil. Nuevamente, demostrando que la certificación de los usuarios corresponde a un proceso eficiente de acuerdo a la opinión de la mayoría de las personas que sí adquirieron el certificado.

El objetivo "Determinar los elementos que motivan al usuario a obtener certificación de cursos en línea en Educación Superior" fue alcanzado con los resultados obtenidos en la sección de "Evaluación y Certificación". Se puede afirmar que el $20 \%$ de los usuarios adquirieron los certificados, mientras que el $80 \%$ restante no lo hicieron debido a las diversas razones explicadas anteriormente. Se obtuvo una amplia visión sobre los motivos que los impulsaron a no certificarse, lo cual sirve como insumo importante para la plataforma, ya que así podrán realizar los ajustes que estén a su alcance, con el fin de promover la certificación de los usuarios. 


\section{REFERENCIAS BIBLIOGRÁFICAS}

Arias, F. (2006). El proyecto de investigación: Introducción a la metodología científica. Caracas: Episteme.

Bernández, M. (2007). Diseño, Producción e Implementación de elearning. Recuperado de https://books.google.co.ve/books?isbn=1434321088

Buendía, L., Colás, P. y Hernández, F. (2001). Métodos de investigación en Psicopedagogía. Madrid: McGraw-Hill. Recuperado de www.ugr.es/ ugr unt/Material\%20M\%F3dulo\%201/variables.pdf

Cabero, J. (2006). Bases pedagógicas del e-learning. RUSC. Universities and Knowledge Society Journal, 3(1). Recuperado de http://rusc.uoc.edu/rusc/es/index.php/rusc/article/download/v3n1-cabero/2651182-2-PB.pdf

Castañón, N. y Del Valle, M. (2015). Proyecto Unimet en Línea: Plan Estratégico para la creación del campus virtual y producción de MOOCs. Recuperado de www.redalyc.org/html/310/31045568014/

Cauas, D. (s.f.). Definición de las variables, enfoque y tipo de investigación. Recuperado de www.mecanicahn.com/personal/marcosmartinez/seminario1/los pdf/Variables.pdf

Cerda, H. (1991). Los elementos de la Investigación: cómo reconocerlos, diseñarlos y construirlos. Bogotá: El Búho. Recuperado de http://postgrado.una.edu.ve/metodologia2/paginas/cerda7.pdf

Dans, E. (2009). Educación online: plataformas educativas y el dilema de la apertura. RUSC. Universities and Knowledge Society Journal, 6(1), 9. Recuperado de http://rusc.uoc.edu/index.php/rusc/article/viewFile/26/21

Dzul, M. (2013). Aplicación básica de los métodos científicos: Diseño NoExperimental. Recuperado de www.uaeh.edu.mx/docencia/VI Presentaciones/licenciatura en mercadotecnia/fu ndamentos de metodologia investigacion/PRES38.pdf

EdX (s.f.). About us. Recuperado de www.edx.org/about-us

Fernández-Valmayor, A., Cristobal, J., Navarro, A., Fernández, A., Merino, J., Peralta, M. y Roldán, Y. (2008). El Campus virtual de la Universidad complutense de Madrid. Pixel-Bit. Revista de Medios y Educación, (32), 55-65. Recuperado de https://idus.us.es/xmlui/handle/11441/45660

García, F., Portillo, J., Romo, J. y Benito, M. (2007). Nativos digitales y modelos de aprendizaje. SPDECE. Recuperado de ftp.informatik.rwth-aachen.de/Publications/CEURWS/Vol-318/Garcia.pdf 
Gómez, T. (2014). Los MOOCs y su papel en la educación universitaria. Recuperado de www.inaecu.com/55736/

Hernández, R., Fernández, C. y Baptista P. (2003). Metodología de la investigación. México D.F.: McGraw-Hil.

Kennard, J. (3 de marzo de 2015). Insight: Nearly half of young people want better business skills. Recuperado de www.trainingzone.co.uk/news/insight-nearly-halfyoung-people-believe-they-need-better-business-skills

Kerlinger, F. (1979) Investigación experimental y no experimental. En: Enfoque conceptual de la investigación del comportamiento. México D.F.: Nueva Editorial Interamericana.

Mendoza, R. (2006). Investigación cualitativa y cuantitativa: Diferencias y limitaciones. Recuperado de www.monografias.com/trabajos38/investigacioncualitativa/investigacion-cualitativa.shtml

Moodle (2015). Moodle statistics. Recuperado de http://moodle.net/stats/

Román, E. (2000). El desarrollo de cursos a distancia en la World Wide Web mediante plataformas virtuales: «WebCT» en el mundo universitario norteamericano. Recuperado de http://e-spacio.uned.es/fez/eserv/bibliuned:1457/n03romanmen00.pdf

Torres, M., Paz, K., y Salazar, F. (s.f.) Métodos de recolección de datos para una investigación. Facultad de Ingeniería Universidad Rafael Landívar. Boletín electrónico, 3(3). Recuperado de www.academia.edu/17287432/URL 03 BAS01

Silvio, J. (2004). Tendencias de la Educación Superior virtual en América Latina y el Caribe. IESALC/UNESCO. Recuperado de www.iesalc.unesco.org.ve/index.php?option $=$ com fabrik\&view=details\&formid $=2 \&$ rowid $=43$

Suárez, C. (2016). Call for paper: Universidad Red desde Iberoamérica. EDMETIC, 5(2), 3-6. Recuperado de http://educacion-virtualidad.blogspot.com/2015/12/call-for-paper-universidad-reddesde.html

Sweeney, S. (2015). Aurion Learning Launch Second Annual Corporate Social Responsibility Report. Recuperado de www.aurionlearning.com/news/aurion-learning-launch-second-annual-corporatesocial-responsibility-report.aspx

Tamayo, T., y Tamayo, M. (1997). El proceso de la investigación científica. México: Editorial Limusa S.A.

Universidad Andrés Bello (s.f.) Tema: Las Variables. Recuperado de http://mey.cl/apuntes/variablesunab.pdf 
Van Dalen, D., y Meyer, W. (2006). La investigación descriptiva. Recuperado de http://noemagico.blogia.com/2006/091301-la-investigaciondescriptiva.php

Van Dusen, G. (1997). The Virtual Campus. ASHE-ERIC Higher Education Report, 25(5).

Vegas, E., Calderón R. \& Rodríguez, R. (2005). El Análisis Cualitativo. Entretemas, 5(3), 1-13. Recuperado de

http://revistas.upel.edu.ve/index.php/entretemas/article/view/1057/378 


\section{CURRÍCULUM VITAE}

Natalia Castañón Octavio

Nacionalidad: venezolana/española

Fecha de nacimiento: 19 de noviembre de 1967

Teléfonos/ e-mail: +584143269864/ nataliacastanon@gmail.com

Trabajo actual: Profesor/investigador de la Universidad Metropolitana (1993- ). Fundadora y directora de tuterapiaenlinea.com (2014- )

Educación superior: Licenciatura en Educación (Universidad Metropolitana, 1991), Maestría en Psicología Escolar (Universidad Complutense de Madrid, 1993), Doctorado en Filosofía y Ciencias de la Educación (Universidad Complutense de Madrid, 1999).

Responsabilidades Académicas: Profesora a tiempo completo de la Universidad Metropolitana (1993-), jefe del departamento de didáctica (2002-), jefe del departamento de humanidades (2014-), gerente general del centro de aprendizaje en línea (2014), profesor investigador.

Productos Académicos: Tutora de treinta (37) tesis de pregrado, maestría y doctorado. Publicaciones en catorce (22) revistas o libros arbitrados a nivel nacional e internacional. Participación en treinta y cuatro (40) congresos Nacionales e Internacionales de ponencias arbitradas. Asesora y/o consultora de más de cuarenta (40) proyectos educativos nacionales e internacionales (Banco Mundial, IBM, PDVSA, Dividendo Voluntario para la Comunidad, Procter \& Gamble, Sanitarios Maracay, Universidad Metropolitana, Fundación Cisneros, Alcaldía de Chacao, entre otros). Líder de 5 proyectos de investigación, nacionales e internacionales, (Conicit, Programa Alfa de la Comunidad Europea, Universidad Metropolitana, entre otros).

Proyectos profesionales: Trabajar en la educación superior, específicamente dos áreas: formación de estudiantes e investigación. Son varios ejes de interés: emprendimiento (plan estratégico incubación de empresas y solución a problemas sociales), cultura de paz (educación al desarme, educación para la democracia, educación derechos humanos, etc.), redes sociales (tecnología y educación) y análisis del contexto (literatura, arte, filosofía, historia y pedagogía), universidad virtual. 